\title{
Tunneling of Massive Vector Particles under the Influence of Quantum Gravity
}

\author{
Wajiha Javed, ${ }^{1, *}$ Riasat Ali, $^{2,+}$ Rimsha Babar, $^{1, \ddagger}$ and Ali Övgün ${ }^{3,4, \S}$ \\ ${ }^{1}$ Division of Science and Technology, University of Education, Township-Lahore, Pakistan \\ ${ }^{2}$ Department of Mathematics, Government College, University Faisalabad Layyah Campus, Layyah-31200, Pakistan \\ ${ }^{3}$ Instituto de Física, Pontificia Universidad Católica de Valparaíso, Casilla 4950, Valparaíso, Chile \\ ${ }^{4}$ Physics Department, Faculty of Arts and Sciences, Eastern Mediterranean University, Famagusta, North Cyprus, via Mersin 10, Turkey
}

(Dated: July 11, 2019)

\begin{abstract}
This paper is devoted to investigate charged vector particles tunneling via horizons of a pair of accelerating rotating charged NUT black hole under the influence of quantum gravitational effects. For this purpose, we use the modified Proca equation incorporating generalized uncertainty principle. Using the WKB approximation to the field equation, we obtain a modified tunneling rate and the corresponding corrected Hawking temperature for this black hole. Moreover, we analyze the graphical behavior of corrected Hawking temperature $T_{H}^{\prime}$ with respect to the event horizon for the given black hole. By considering quantum gravitational effects on Hawking temperatures, we discuss the stability analysis of this black hole. For a pair of black holes, the temperature $T_{H}^{\prime}$ increases with the increase in rotation parameters $a$ and $\omega$, correction parameter $\beta$, black hole acceleration $\alpha$ and arbitrary parameter $k$ and decreases with the increase in electric $e$ and magnetic charges $g$.
\end{abstract}

PACS numbers: 04.70.Dy; 04.70.Bw; 04.60.-m

Keywords: Massive boson particles; Wave equation; quantum gravity; Hawking radiation; Hawking radiation; Black hole thermodynamics

\section{INTRODUCTION}

A black hole $(\mathrm{BH})$ is a physical object that has the ability to absorb all types of energy from the surrounding due to its strong gravitational pull. According to the theory of general relativity, a $\mathrm{BH}$ attracts all types of particles that interact with event horizon. Hawking (1974) described that, a BH acts as a black body and emit particles in form of radiation through its horizon by considering quantum effects in the background of curved spacetime, these radiation are known as Hawking radiation [1] and possesses a particular temperature which is called Hawking temperature. In order to study the Hawking radiation phenomenon, the quantum tunneling is one of the best technique [2]-[4]. This technique depends on electron-positron pair-creation, which needs an electric field. It could be a well-known fact that as particles overcome the horizon, the energy changes the sign, so that a pair created just inside/outside the horizon can materialize with zero total energy, while one member of the pair has ability to tunnel to the opposite side of horizon [5].

According to the tunneling phenomenon, particles are permitted to follow the classically forbidden trajectories, by starting from outside the horizon to infinity [6,7]. The BH evaporation can be studied with the discharge of quantum particles in form of Hawking radiation which enables BH to lose its mass. When a BH loses more matter instead of increasing through different means then it disseminate, shrink and eventually disappears. This phenomenon causes a change in thermodynamical characteristic of a $\mathrm{BH}$, i.e., charge, mass and angular momentum. These particles follow the frame of outgoing/ingoing radial null geodesics. For the outgoing geodesics, the particles must be imaginary although for ingoing geodesics, they are considered to be real. It is due to the fact that just a real particle that has a speed not exactly or equivalent to the speed of light can exist inside the horizon.

For calculating the imaginary part of the classical action there are two main approaches, i.e., null geodesic technique and Hamilton-Jacobi strategy. The first one proposed by Parikh and his colleagues [5] and second one introduced by Srinivasan and Padmanabhan [6]. To construct a relation between classical and quantum theory, an approximation is known as WKB approximation utilized by Wentzel, Kramers and Brillouin (WKB) [8]. Various authors studied tunneling of vector particles, bosonic particles, spin-2, spin-3/2 and fermionic particles to obtain the Hawking temperature for different $\mathrm{BHs}$ and wormholes [9-59].

\footnotetext{
*Electronic address: wajiha.javed@ue.edu.pk; wajihajaved84@yahoo.com

†Electronic address: riasatyasin@gmail.com

‡Electronic address: rimsha.babar10@gmail.com

§Electronic address: ali.ovgun@pucv.cl; https://www.aovgun.com
} 
By incorporating generalized uncertainty principle (GUP) effects, it is conceivable to discuss quantum corrected thermodynamical properties of $\mathrm{BH}$ [60]. The GUP gives high-energy remedies to $\mathrm{BH}$ thermodynamics, which leads to the possibility of a minimal length in quantum gravity theory. The idea of GUP has been utilized for various BHs. The tunneling process for Kerr, Kerr-Newman and Reissner-Nordström (RN) BHs [61] provides important contribution towards the BH physics. Jiang [62] calculated the tunneling of Dirac particles and analyzed the Hawking radiation spectrum for black ring. Jian and Bing-Bing [63] studied the fermion particles tunneling from uncharged and charged BHs. Yale [64] studied the scalar, fermion and boson particles tunneling from BHs without back-reaction effects, they also analyzed the exact Hawking temperature for these particles. Sharif and Javed [65] studied the Hawking radiation through quantum tunneling process for various types of BHs and also derived the tunneling probabilities as well as their corresponding Hawking temperatures. The same authors [66] calculated the tunneling behavior of fermion particles from traversable wormholes. They also discussed the tunneling behavior of fermion particles for charged accelerating rotating BHs associated with NUT parameter [67].

Övgün et al. [68] calculated the tunneling rate of charged massive bosons for various types of BHs surrounded by the perfect fluid in Rastall theory. Anacleto et al. [69] studied the finite quantum corrected entropy from noncommutative acoustic BHs. Li and Zu [70] analyzed the GUP effects by utilizing Klein-Gordon equation and also studied corrected temperature for Gibbon-Maeda-Dilation BH. Anacleto et al. [71] investigated the quantum corrected entropy by using tunneling method with GUP in self-dual BH. Övgün and Jusufi [72] studied the spin-1 vector particles tunneling for charged non-commutative BHs and its GUP-corrected thermodynamical properties. Singh et al. [73] analyzed particles radiation for Kerr-Newman BH by using quantum tunneling phenomenon. Sakalli et al. [74] investigated the scalar particles tunneling from acoustic BHs with a rotation parameter by applying $W K B$ approximation and Klein-Gordon equation with GUP to obtain Hawking temperature of massive particles.

Javed et al. [75] analyzed charged vector particles tunneling phenomenon for a pair of accelerating and rotating as well as 5D gauged super-gravity BHs and investigated the corresponding Hawking temperatures. In the continuation of their work, we study the Hawking radiation phenomenon by considering quantum corrections of boson particles through the event horizon of accelerating and rotating BH with NUT parameter. We also discuss the graphical behavior of corrected temperature with respect to horizon for given $\mathrm{BH}$ and analyzed the effects of different parameters on temperature and visualize the stable and unstable states of BH. The main motivation of this paper is to study the charged accelerating and rotating black hole with NUT parameters under the influence of quantum gravity effects and to check how the results reduce to the previous results when we neglect the quantum gravity effects as well as other parameters. The organization of this paper is as follows: In section 2, we study the quantum corrected tunneling rate and corrected Hawking temperature for charged $\mathrm{BH}$ solution having acceleration, rotation and NUT parameter. Moreover, we analyze the graphical behavior of corrected temperature with respect to the event horizon of $\mathrm{BH}$ and also discuss the effects of different parameters on temperature. Section 3 is based on the conclusion and discussion of the mathematical results for these BHs.

\section{PAIR OF CHARGED ACCELERATED BLACK HOLES INVOLVING ROTATION AND NUT PARAMETERS}

Universally, the NUT parameter is associated with the twisting behavior of the surrounding spacetime or with the gravito-magnetic monopole parameter of the central mass. Moreover, its precise physical significance might not be investigated. Later, the higher dimensional origin of the Kerr-NUT-(anti) de-Sitter BH and its physical significance is studied [76,77]. For a BH, the apparent dominance of the NUT parameter on the rotation parameter takes off the metric free of curvature singularity and the corresponding result is known as NUT-like result. When the rotation parameter commands the NUT parameter, the solution is Kerr-like and a ring curvature singularity occurs. This type of singularity is independent from existence of the cosmological constant.

The exact understanding of the NUT parameter is conceivable when a static Schwarzschild mass is submerged in a stationary source-free electromagnetic universe [78]. Moreover, the NUT parameter is associated with the representation of twisting behavior of the electromagnetic universe allowing off the Schwarzschild central mass. When electric and magnetic fields vanish, the NUT parameter represents the twist of the vacuum spacetime [67]. Consequently, for creation of NUT parameter, the bending of the environmental space couples with the mass of background field.

The line-element of this $\mathrm{BH}$ can be defined as follows [79]

$$
\begin{aligned}
d s^{2}= & -\frac{1}{\Omega^{2}}\left[-\frac{Q}{\rho^{2}}\left(\left(4 l \sin ^{2} \frac{\theta}{2}+a \sin ^{2} \theta\right) d \phi-d t\right)^{2}-\frac{\rho^{2}}{Q} d r^{2}\right. \\
& \left.+\frac{\tilde{P}}{\rho^{2}}\left(\left((l+a)^{2}+r^{2}\right) d \phi-a d t\right)^{2}-\sin ^{2} \theta \frac{\rho^{2}}{\tilde{P}} d \theta^{2}\right]
\end{aligned}
$$


where

$$
\begin{aligned}
\Omega & =-(a \cos \theta+l) \frac{\alpha}{\omega} r+1, \quad \rho^{2}=(a \cos \theta+l)^{2}+r^{2} \\
Q & =\left[\left(\tilde{e}^{2}+\omega^{2} \tilde{k}+\tilde{g}^{2}\right)\left(1+2 l \alpha \frac{r}{\omega}\right)-2 r M-\frac{\omega^{2} \tilde{k} r^{2}}{l^{2}-a^{2}}\right] \\
& \times\left[1-\frac{l-a}{\omega} \alpha r\right]\left[1-\frac{l+a}{\omega} r \alpha\right], \\
\tilde{P} & =-\sin ^{2} \theta\left(a_{4} \cos ^{2} \theta+a_{3} \cos \theta-1\right)=\sin ^{2} \theta P, \\
a_{3} & =\frac{\alpha a}{\omega} 2 M-4 \frac{\alpha^{2} a l}{\omega^{2}}\left(\tilde{g}^{2}+\tilde{e}^{2}+\omega^{2} \tilde{k}\right), \\
a_{4} & =-\frac{\alpha^{2} a^{2}}{\omega^{2}}\left(\tilde{g}^{2}+\tilde{e}^{2}+\omega^{2} \tilde{k}\right)
\end{aligned}
$$

where $M, g$ and $e$ represent mass, magnetic and electric charges, respectively. While $l, \omega$ and $\alpha$ are NUT parameter, rotation and acceleration of source, respectively. Moreover, $a$ is rotation parameter (parameter of Kerr-like) and $\tilde{k}$ is defined as

$$
\left(\frac{\omega^{2}}{l^{2}-a^{2}}-3 \alpha^{2} l^{2}\right) \tilde{k}=3 \frac{\alpha^{2} l^{2}}{\omega^{2}}\left(\tilde{e}^{2}+\tilde{g}^{2}\right)-2 \frac{\alpha l}{\omega} M-1 .
$$

We observe that $\omega$ depends on $a$ and $l$. The parameter $\alpha$ represents the bending behavior of BHs and relative to the rotation parameter $(\omega)$. The parameters $\tilde{e}, \tilde{g}, M, \omega, \tilde{k}$ and $\alpha$ are independent. As $\alpha=0$, then the line-element (1) implies to the Kerr-Newman NUT results. For $l=0$, the line-element (1) gives the BHs with rotating and charged parameters. When $\tilde{g}$ and $\tilde{e}$ approaches to zero then we get a Schwarzschild $\mathrm{BH}$ and if $a$ and $l$ approaches to zero, then it implies to the C-metric.

The line-element (1) can be rewritten in the following form

$$
d s^{2}=-Z(r, \theta) d t^{2}+\frac{d r^{2}}{B(r, \theta)}+C(r, \theta) d \theta^{2}+D(r, \theta) d \phi^{2}-2 F(r, \theta) d \phi d t,
$$

where the metric functions $Z, B, C, D$ and $F$ are given as follows

$$
\begin{aligned}
& Z(r, \theta)=\frac{-P a^{2} \sin ^{2} \theta+Q}{\Omega^{2} \rho^{2}}, \quad B(r, \theta)=\frac{\Omega^{2} Q}{\rho^{2}}, \quad C(r, \theta)=\frac{\rho^{2}}{P \Omega^{2}} \\
& D(r, \theta)=\frac{1}{\rho^{2} \Omega^{2}}\left(\sin ^{2} \theta Q\left((a+l)^{2}+r^{2}\right)^{2}-Q\left(4 l \sin ^{2} \frac{\theta}{2}+a \sin ^{2} \theta\right)^{2}\right) \\
& F(r, \theta)=\frac{1}{\rho^{2} \Omega^{2}}\left(P a \sin ^{2} \theta\left((l+a)^{2}+r^{2}\right)-Q\left(4 l \sin ^{2} \frac{\theta}{2}+a \sin ^{2} \theta\right)\right) .
\end{aligned}
$$

The electromagnetic potential for these pair of $\mathrm{BHs}$ is defined as

$$
\begin{aligned}
A & =\frac{1}{a\left((a \cos \theta+l)^{2}+r^{2}\right)}\left[\tilde { g } \left(-l-a \cos \theta\left(a d t-\left(r^{2}+(a+l)^{2}\right) d \phi\right.\right.\right. \\
& \left.+r \tilde{e}\left(d \phi\left((a+l)-a d t+\left(2 a l \cos \theta+a^{2} \cos ^{2} \theta+l^{2}\right)\right)\right)\right] .
\end{aligned}
$$

The horizons are obtained from $B(r, \theta)=\frac{\Omega^{2} Q}{\rho^{2}}=0$, which implies that $\Omega$ is not zero, thus $Q$ is zero, so $r$ has real roots, i.e.,

$$
\begin{aligned}
& r_{\alpha 1}=\frac{\omega}{\alpha(l+a)}, \quad r_{\alpha 2}=-\frac{\omega}{(a-l) \alpha}, \quad r_{ \pm}=\frac{l^{2}-a^{2}}{\omega^{2} \tilde{k}}\left[\left(\left(\tilde{g}^{2}+\tilde{e}^{2}+\omega^{2} \tilde{k}\right) \frac{\alpha l}{\omega}\right.\right. \\
& -M) \pm \sqrt{\left.\left(\left(\tilde{g}^{2}+\tilde{e}^{2}+\omega^{2} \tilde{k}\right) \frac{\alpha l}{\omega}-M\right)^{2}+\left(\tilde{g}^{2}+\tilde{e}^{2}+\omega^{2} \tilde{k}\right) \frac{\omega^{2} \tilde{k}}{l^{2}-\alpha^{2}}\right]} .
\end{aligned}
$$

Here, $r_{\alpha 1}$ and $r_{\alpha 2}$ represent the acceleration horizons and $r_{ \pm}$denote the inner and outer horizons such that

$$
\left(\left(\tilde{g}^{2}+\tilde{e}^{2}+\omega^{2} \tilde{k}\right) \frac{\alpha l}{\omega}-M\right)^{2}-\left(\tilde{g}^{2}+\tilde{e}^{2}+\omega^{2} \tilde{k}\right) \frac{\omega^{2} \tilde{k}}{a^{2}-l^{2}}>0 .
$$


The angular velocity of $\mathrm{BH}$ at outer horizon is given as follows

$$
\Omega_{H}=\frac{a}{(a+l)^{2}+r_{+}^{2}} .
$$

In order to study the corrected tunneling rate for boson particles through the pair of BH horizon, we consider Lagrangian equation with quantum gravity effects. By considering a spacetime with potential due to both electric and magnetic field, the behavior of massive (spin-1) boson is described by the wave equation. The GUP modified Lagrangian equation with vector field $\psi_{\mu}$ for bosons particles can be expressed as [80, 81]

$$
£^{G U P}=-\frac{1}{2}\left(\mathfrak{D}_{\mu} \psi_{\nu}-\mathfrak{D}_{\nu} \psi_{\mu}\right)\left(\mathfrak{D}^{\mu} \psi^{\nu}-\mathfrak{D}^{v} \psi^{\mu}\right)-\frac{\iota}{\hbar} e F^{v \mu} \psi_{\mu} \psi_{v}-\frac{m^{2}}{\hbar^{2}} \psi_{\mu} \psi^{v} .
$$

The modified wave equation for massive bosons can be defined as follows

$$
\begin{aligned}
& \partial_{\mu}\left(\sqrt{-g} \psi^{\nu \mu}\right)+\sqrt{-g} \frac{m^{2}}{\hbar^{2}} \psi^{\nu}+\sqrt{-g} \frac{\iota}{\hbar} e A_{\mu} \psi^{v \mu}+\sqrt{-g} \frac{\iota}{\hbar} e F^{v \mu} \psi_{\mu}+\beta \hbar^{2} \partial_{0} \partial_{0} \partial_{0} \\
& \left(\sqrt{-g} g^{00} \psi^{0 v}\right)-\beta \hbar^{2} \partial_{i} \partial_{i} \partial_{i}\left(\sqrt{-g} g^{i i} \psi^{i v}\right)=0,
\end{aligned}
$$

where $|g|, m$ and $\psi^{\mu v}$ are the matrix coefficients, mass of a particle and anti-symmetric tensor respectively. The anti-symmetric tensor can be defined as

$$
\begin{gathered}
\psi_{\nu \mu}=\left(1-\beta \hbar^{2} \partial_{\nu}^{2}\right) \partial_{\nu} \psi_{\mu}-\left(1-\beta \hbar^{2} \partial_{\mu}^{2}\right) \partial_{\mu} \psi_{v}+\left(1-\beta \hbar^{2} \partial_{v}^{2}\right) \frac{\iota}{\hbar} e A_{\nu} \psi_{\mu} \\
-\left(1-\beta \hbar^{2} \partial_{\mu}^{2}\right) \frac{\iota}{\hbar} e A_{\mu} \psi_{\nu}, \text { and } \\
F_{\mu \nu}=\nabla_{\mu} A_{v}-\nabla_{\nu} A_{\mu}, \text { where } \nabla_{0}=\left(1+\beta \hbar^{2} g^{00} \nabla_{0}^{2}\right) \nabla_{0}, \nabla_{0}=\left(1-\beta \hbar^{2} g^{i i} \nabla_{i}^{2}\right) \nabla_{i},
\end{gathered}
$$

where $\beta$ is the representation of the quantum gravity effect in terms of correction parameter, if $\beta=0$ and $A=0$, then the above wave equation reduces to the expressions given in Ref.[75] and Ref.[68], respectively, if both $\beta$ and $A$ are zero then this wave equation reduces to the wave equation given in Ref.[73]. While, $A_{\mu}$ is considered as the $\mathrm{BH}$ electromagnetic potential, $e$ denotes the bosons particles charge and $\nabla_{\mu}$ is representing covariant derivative. In the equation of wave motion the +ive and -ive bosons have alike behavior, the quantum tunneling phenomena for both type of particles is also similar. For + ive field, the values of $\psi^{\mu}$ and $\psi^{\nu \mu}$ are obtained as follows

$$
\begin{aligned}
& \psi^{0}=\frac{-D \psi_{0}-F \psi_{3}}{Z D+F^{2}}, \quad \psi^{1}=B \psi_{1}, \quad \psi^{2}=C^{-1} \psi_{2}, \quad \psi^{3}=\frac{-F \psi_{0}+Z \psi_{3}}{Z D+F^{2}} \\
& \psi^{01}=\frac{-B D \psi_{01}-B F \psi_{13}}{Z D+F^{2}}, \quad \psi^{02}=\frac{-D \psi_{02}-F \psi_{23}}{C\left(Z D+F^{2}\right)}, \quad \psi^{03}=\frac{-\psi_{03}}{Z D+F^{2}} \\
& \psi^{12}=B C^{-1} \psi_{12}, \quad \psi^{13}=\frac{B\left(Z \psi_{13}-F \psi_{01}\right)}{Z D+F^{2}}, \quad \psi^{23}=\frac{B \psi_{23}-F \psi_{02}}{C\left(Z D+F^{2}\right)}
\end{aligned}
$$

Utilizing WKB approximation [82]

$$
\psi_{v}=c_{v} \exp \left[\frac{\iota}{\hbar} \tilde{I}_{0} \alpha_{n}+\Sigma \hbar^{n} \tilde{I}_{n} \alpha_{n}\right]
$$

where $\alpha_{n}=(t, r, \theta, \phi)$ (for $\left.n=1,2,3,4\right)$ to the Lagrangian wave Eq.(6) and ignoring the higher terms, one can obtain 
the following set of equations

$$
\begin{aligned}
& D B\left[c_{1}\left(\partial_{0} \tilde{I}_{o}\right)\left(\partial_{1} \tilde{I}_{o}\right)-c_{1} \beta\left(\partial_{0} \tilde{I}_{o}\right)^{3}\left(\partial_{1} \tilde{I}_{o}\right)+c_{1} \beta\left(\partial_{1} \tilde{I}_{o}\right)\left(\partial_{0} \tilde{I}_{o}\right)^{2} e A_{0}\right. \\
& \left.-c_{0} \beta\left(\partial_{1} \tilde{I}_{o}\right)^{4}+e A_{0} c_{1}\left(\partial_{1} \tilde{I}_{o}\right)-c_{0}\left(\partial_{1} \tilde{I}_{o}\right)^{2}\right]+B F\left[c_{3}\left(\partial_{1} \tilde{I}_{o}\right)^{2}+c_{3} \beta\left(\partial_{1} \tilde{I}_{o}\right)^{3}\right. \\
& \left.-c_{1}\left(\partial_{1} \tilde{I}_{o}\right)\left(\partial_{3} \tilde{I}_{0}\right)-c_{1} \beta\left(\partial_{3} \tilde{I}_{o}\right)^{3}\left(\partial_{1} \tilde{I}_{0}\right)-e A_{3} c_{1} \beta\left(\partial_{3} \tilde{I}_{o}\right)^{2}\left(\partial_{1} \tilde{I}_{0}\right)\right]+D C^{-1} \\
& \times \quad c_{2} \beta e A_{0}\left(\partial_{0} \tilde{I}_{o}\right)^{2}\left(\partial_{2} \tilde{I}_{0}\right)+c_{2} \beta\left(\partial_{0} \tilde{I}_{o}\right)^{3}\left(\partial_{2} \tilde{I}_{0}\right)-c_{0} \beta\left(\partial_{2} \tilde{I}_{o}\right)^{2}-c_{0} \beta\left(\partial_{2} \tilde{I}_{o}\right)^{4} \\
& \left.+c_{2} e A_{0}\left(\partial_{2} \tilde{I}_{0}\right)+c_{2}\left(\partial_{0} \tilde{I}_{0}\right)\left(\partial_{2} \tilde{I}_{0}\right)\right]+F^{-1}\left[c_{3}\left(\partial_{2} \tilde{I}_{0}\right)^{2}+c_{3} \beta\left(\partial_{2} \tilde{I}_{o}\right)^{4}\right. \\
& -c_{2}\left(\partial_{3} \tilde{I}_{o}\right)\left(\partial_{2} \tilde{I}_{o}\right)-c_{2} \beta\left(\partial_{2} \tilde{I}_{o}\right)\left(\partial_{3} \tilde{I}_{o}\right)^{3}+c_{2} e A_{3}\left(\partial_{2} \tilde{I}_{o}\right)+c_{2} e A_{3} \beta\left(\partial_{3} \tilde{I}_{o}\right)^{2} \\
& \left.\times\left(\partial_{2} \tilde{I}_{0}\right)\right]+\left[c_{3}\left(\partial_{3} \tilde{I}_{0}\right)\left(\partial_{0} \tilde{I}_{o}\right)-c_{3} \beta\left(\partial_{0} \tilde{I}_{0}\right)^{3}\left(\partial_{3} \tilde{I}_{0}\right)-c_{0}\left(\partial_{3} \tilde{I}_{o}\right)^{2}-c_{0} \beta\left(\partial_{3} \tilde{I}_{0}\right)^{4}\right. \\
& \left.-c_{0} e A_{3}\left(\partial_{3} \tilde{I}_{o}\right)-c_{0} e A_{3} \beta\left(\partial_{3} \tilde{I}_{o}\right)^{3}+c_{3} e A_{0}\left(\partial_{3} \tilde{I}_{o}\right)+c_{3} e A_{0} \beta\left(\partial_{0} \tilde{I}_{o}\right)^{2}\left(\partial_{3} \tilde{I}_{o}\right)\right] \\
& +e A_{3}\left[c_{3}\left(\partial_{0} \tilde{I}_{o}\right)+c_{3} \beta\left(\partial_{0} \tilde{I}_{o}\right)^{3}-c_{0}\left(\partial_{3} \tilde{I}_{o}\right)+c_{0} \beta\left(\partial_{3} \tilde{I}_{o}\right)^{3}-c_{3} e A_{0}\right. \\
& \left.-c_{3} \beta\left(\partial_{0} \tilde{I}_{0}\right)^{2}+c_{0} e A_{3}+c_{0} e A_{3} \beta\left(\partial_{3} \tilde{I}_{0}\right)^{2}\right]-D m^{2} c_{0}-F m^{2} c_{3}=0, \\
& D \quad\left[c_{1}\left(\partial_{0} \tilde{I}_{o}\right)^{2}+c_{1} \beta\left(\partial_{0} \tilde{I}_{o}\right)^{4}-c_{0}\left(\partial_{0} \tilde{I}_{o}\right)\left(\partial_{1} \tilde{I}_{o}\right)+c_{0} \beta\left(\partial_{0} \tilde{I}_{o}\right)\left(\partial_{1} \tilde{I}_{o}\right)^{3}\right. \\
& \left.-e A_{0} c_{1}\left(\partial_{0} \tilde{I}_{o}\right)+c_{1} \beta\left(\partial_{0} \tilde{I}_{o}\right)^{3} e A_{0}\right]+F\left[c_{3}\left(\partial_{0} \tilde{I}_{o}\right)\left(\partial_{1} \tilde{I}_{o}\right)+c_{3} \beta\left(\partial_{1} \tilde{I}_{o}\right)^{3}\left(\partial_{0} \tilde{I}_{o}\right)\right. \\
& \left.-c_{1}\left(\partial_{0} \tilde{I}_{o}\right)\left(\partial_{3} \tilde{I}_{o}\right)-c_{1} \beta\left(\partial_{3} \tilde{I}_{o}\right)^{3}\left(\partial_{0} \tilde{I}_{o}\right)-c_{1} e A_{3}\left(\partial_{0} \tilde{I}_{o}\right)-e A_{3} c_{1} \beta\left(\partial_{3} \tilde{I}_{o}\right)^{2}\left(\partial_{0} \tilde{I}_{o}\right)\right] \\
& +\left(Z D+F^{2}\right) C^{-1}\left[c_{2}\left(\partial_{1} \tilde{I}_{o}\right)\left(\partial_{2} \tilde{I}_{o}\right)+c_{2} \beta\left(\partial_{1} \tilde{I}_{o}\right)^{3}\left(\partial_{2} \tilde{I}_{o}\right)-c_{1} \beta\left(\partial_{2} \tilde{I}_{o}\right)^{2}\right. \\
& \left.-c_{1} \beta\left(\partial_{2} \tilde{I}_{o}\right)^{4}\right]-m^{2} c_{1}+e A_{0} D\left[c_{1}\left(\partial_{0} \tilde{I}_{o}\right)-c_{1} \beta\left(\partial_{0} \tilde{I}_{o}\right)^{3}-c_{0}\left(\partial_{1} \tilde{I}_{o}\right)\right. \\
& \left.-c_{0} \beta\left(\partial_{1} \tilde{I}_{0}\right)^{3}+c_{1} e A_{0}+c_{1} e A_{0} \beta\left(\partial_{0} \tilde{I}_{o}\right)^{2}\right]+e A_{0} F\left[c_{3}\left(\partial_{1} \tilde{I}_{o}\right)+c_{3} \beta\left(\partial_{0} \tilde{I}_{o}\right)^{3}\right. \\
& \left.-c_{1}\left(\partial_{3} \tilde{I}_{o}\right)-c_{0} \beta\left(\partial_{3} \tilde{I}_{o}\right)^{3}-c_{1} e A_{3}-c_{1} e A_{3} \beta\left(\partial_{3} \tilde{I}_{o}\right)^{2}\right]+e A_{0} A\left[c_{3}\left(\partial_{1} \tilde{I}_{o}\right)\right. \\
& \left.+c_{3} \beta\left(\partial_{1} \tilde{I}_{o}\right)^{3}-c_{1}\left(\partial_{3} \tilde{I}_{o}\right)-c_{1} \beta\left(\partial_{3} \tilde{I}_{o}\right)^{3}-c_{1} e A_{3}-c_{1} \beta\left(\partial_{3} \tilde{I}_{o}\right)^{2} e A_{3}\right]
\end{aligned}
$$




$$
\begin{aligned}
& +e A_{3} F\left[c_{1}\left(\partial_{0} \tilde{I}_{o}\right)+c_{1} \beta\left(\partial_{0} \tilde{I}_{o}\right)^{3}-c_{0}\left(\partial_{1} \tilde{I}_{o}\right)-c_{0} \beta\left(\partial_{1} \tilde{I}_{o}\right)^{3}+c_{1} e A_{0}\right. \\
& \left.+c_{1} \beta\left(\partial_{0} \tilde{I}_{0}\right)^{2} e A_{0}\right]=0 \text {, } \\
& F\left[c_{3}\left(\partial_{0} \tilde{I}_{o}\right)\left(\partial_{2} \tilde{I}_{o}\right)+c_{3} \beta\left(\partial_{0} \tilde{I}_{o}\right)\left(\partial_{2} \tilde{I}_{0}\right)^{3}-c_{2}\left(\partial_{0} \tilde{I}_{o}\right)\left(\partial_{3} \tilde{I}_{o}\right)-c_{2} \beta\left(\partial_{0} \tilde{I}_{o}\right)\right. \\
& \left.\times\left(\partial_{3} \tilde{I}_{o}\right)^{3}-e A_{3} c_{2}\left(\partial_{0} \tilde{I}_{o}\right)-c_{2} \beta\left(\partial_{3} \tilde{I}_{o}\right)^{2}\left(\partial_{0} \tilde{I}_{o}\right) e A_{0}\right]+D\left[c_{2}\left(\partial_{0} \tilde{I}_{o}\right)^{2}\right. \\
& -c_{0}\left(\partial_{0} \tilde{I}_{o}\right)\left(\partial_{2} \tilde{I}_{o}\right)+c_{2} \beta\left(\partial_{0} \tilde{I}_{o}\right)^{4}-c_{0} \beta\left(\partial_{2} \tilde{I}_{o}\right)^{3}\left(\partial_{0} \tilde{I}_{o}\right)+c_{2} e A_{0}\left(\partial_{0} \tilde{I}_{o}\right) \\
& \left.+e A_{3} c_{2} \beta\left(\partial_{2} \tilde{I}_{o}\right)^{2}\left(\partial_{0} \tilde{I}_{o}\right)\right]-B\left[c_{2}\left(\partial_{1} \tilde{I}_{o}\right)^{2}+c_{2} \beta\left(\partial_{1} \tilde{I}_{o}\right)^{4}-c_{1} \beta\left(\partial_{1} \tilde{I}_{o}\right)\left(\partial_{2} \tilde{I}_{o}\right)\right. \\
& \left.-c_{1} \beta\left(\partial_{1} \tilde{I}_{o}\right)\left(\partial_{2} \tilde{I}_{o}\right)^{3}\right]+B\left[c_{3}\left(\partial_{3} \tilde{I}_{o}\right)\left(\partial_{2} \tilde{I}_{o}\right)+c_{3} \beta\left(\partial_{3} \tilde{I}_{o}\right)\left(\partial_{2} \tilde{I}_{o}\right)^{3}-c_{2} \beta\left(\partial_{3} \tilde{I}_{o}\right)^{2}\right. \\
& \left.-c_{2} \beta\left(\partial_{3} \tilde{I}_{o}\right)^{4}-e A_{3} c_{2}\left(\partial_{3} \tilde{I}_{o}\right)-c_{2} \beta\left(\partial_{3} \tilde{I}_{o}\right)^{3} e A_{3}\right]-F\left[c_{2}\left(\partial_{0} \tilde{I}_{o}\right)\left(\partial_{3} \tilde{I}_{o}\right)\right. \\
& +c_{2} \beta\left(\partial_{3} \tilde{I}_{o}\right)\left(\partial_{0} \tilde{I}_{0}\right)^{3}-c_{0} \beta\left(\partial_{2} \tilde{I}_{o}\right)\left(\partial_{3} \tilde{I}_{o}\right)-c_{0} \beta\left(\partial_{2} \tilde{I}_{0}\right)^{3}\left(\partial_{3} \tilde{I}_{o}\right)+e A_{0} c_{2}\left(\partial_{3} \tilde{I}_{o}\right) \\
& \left.+c_{2} \beta\left(\partial_{0} \tilde{I}_{0}\right)^{2}\left(\partial_{3} \tilde{I}_{0}\right) e A_{0}\right]-\left(Z B+F^{2}\right) m^{2} c_{2}+e A_{0} F\left[c_{3}\left(\partial_{2} \tilde{I}_{0}\right)+c_{3} \beta\left(\partial_{2} \tilde{I}_{o}\right)^{3}\right. \\
& \left.-c_{2}\left(\partial_{3} \tilde{I}_{o}\right)-c_{2} \beta\left(\partial_{3} \tilde{I}_{o}\right)^{3}-c_{2} e A_{3}-c_{2} e A_{3} \beta\left(\partial_{3} \tilde{I}_{o}\right)^{2}\right]+e A_{0} D\left[c_{2}\left(\partial_{0} \tilde{I}_{o}\right)\right. \\
& \left.+c_{2} \beta\left(\partial_{0} \tilde{I}_{0}\right)^{3}-c_{0}\left(\partial_{2} \tilde{I}_{0}\right)-c_{0} \beta\left(\partial_{2} \tilde{I}_{o}\right)^{3}+c_{2} e A_{0}+c_{2} e A_{0} \beta\left(\partial_{0} \tilde{I}_{0}\right)^{2}\right] \\
& +e A_{3} B\left[c_{3}\left(\partial_{2} \tilde{I}_{o}\right)+c_{3} \beta\left(\partial_{2} \tilde{I}_{o}\right)^{3}-c_{2}\left(\partial_{3} \tilde{I}_{o}\right)-c_{2} \beta\left(\partial_{3} \tilde{I}_{o}\right)^{3}-c_{2} e A_{3}\right. \\
& \left.-c_{2} \beta e A_{3}\left(\partial_{3} \tilde{I}_{o}\right)^{2}\right]+e A_{3} F\left[c_{2}\left(\partial_{0} \tilde{I}_{o}\right)+c_{2} \beta\left(\partial_{0} \tilde{I}_{o}\right)^{3}-c_{0}\left(\partial_{2} \tilde{I}_{o}\right)-c_{0} \beta\left(\partial_{2} \tilde{I}_{o}\right)^{3}\right. \\
& \left.+c_{2} e A_{0}+c_{2} \beta\left(\partial_{0} \tilde{I}_{0}\right)^{2} e A_{0}\right]=0 \text {, } \\
& F\left[c_{2}\left(\partial_{0} \tilde{I}_{0}\right)\left(\partial_{3} \tilde{I}_{o}\right)+c_{2} \beta\left(\partial_{3} \tilde{I}_{o}\right)\left(\partial_{0} \tilde{I}_{o}\right)^{3}-c_{0} \beta\left(\partial_{3} \tilde{I}_{o}\right)\left(\partial_{2} \tilde{I}_{0}\right)-c_{0} \beta\left(\partial_{2} \tilde{I}_{o}\right)^{3}\right. \\
& \left.\times\left(\partial_{3} \tilde{I}_{0}\right)+e A_{0} c_{2}\left(\partial_{3} \tilde{I}_{0}\right)+c_{2} \beta\left(\partial_{0} \tilde{I}_{0}\right)^{2}\left(\partial_{3} \tilde{I}_{0}\right) e A_{0}\right]\left[c_{3}\left(\partial_{0} \tilde{I}_{o}\right)^{2}+c_{3} \beta\left(\partial_{0} \tilde{I}_{o}\right)^{4}\right. \\
& -c_{0}\left(\partial_{0} \tilde{I}_{o}\right)\left(\partial_{3} \tilde{I}_{o}\right)-c_{0} \beta\left(\partial_{0} \tilde{I}_{o}\right)\left(\partial_{3} \tilde{I}_{o}\right)^{3}-e A_{3} c_{3}\left(\partial_{0} \tilde{I}_{o}\right)+c_{3} \beta\left(\partial_{0} \tilde{I}_{o}\right)^{3} e A_{3} \\
& \left.-c_{0}\left(\partial_{0} \tilde{I}_{o}\right) e A_{3}-c_{0} \beta\left(\partial_{3} \tilde{I}_{o}\right)^{2}\left(\partial_{0} \tilde{I}_{o}\right) e A_{3}\right]+B F\left[c_{1}\left(\partial_{0} \tilde{I}_{o}\right)\left(\partial_{1} \tilde{I}_{o}\right)+c_{1} e A_{0}\right. \\
& \times\left(\partial_{1} \tilde{I}_{o}\right)-c_{0}\left(\partial_{1} \tilde{I}_{o}\right)^{2}-c_{0} \beta\left(\partial_{2} \tilde{I}_{o}\right)^{4}+c_{1} \beta\left(\partial_{0} \tilde{I}_{o}\right)^{3}\left(\partial_{1} \tilde{I}_{o}\right)+e A_{0} c_{1} \beta\left(\partial_{0} \tilde{I}_{o}\right)^{2} \\
& \left.\times\left(\partial_{1} \tilde{I}_{0}\right)\right]-Z B\left[c_{3}\left(\partial_{1} \tilde{I}_{0}\right)^{2}+c_{3} \beta\left(\partial_{1} \tilde{I}_{0}\right)^{4}-c_{1} \beta\left(\partial_{1} \tilde{I}_{o}\right)\left(\partial_{3} \tilde{I}_{o}\right)-c_{1} \beta\left(\partial_{1} \tilde{I}_{o}\right)\right. \\
& \left.\times\left(\partial_{3} \tilde{I}_{o}\right)^{2}-c_{1} e A_{3}\left(\partial_{1} \tilde{I}_{0}\right)+c_{1} \beta e A_{3}\left(\partial_{3} \tilde{I}_{o}\right)^{2}\left(\partial_{1} \tilde{I}_{0}\right)\right]-B\left[c_{3}\left(\partial_{2} \tilde{I}_{o}\right)^{2}\right. \\
& -c_{2}\left(\partial_{3} \tilde{I}_{o}\right)\left(\partial_{2} \tilde{I}_{o}\right)+c_{3} \beta\left(\partial_{2} \tilde{I}_{0}\right)^{4}-c_{2} \beta\left(\partial_{3} \tilde{I}_{o}\right)^{3}\left(\partial_{2} \tilde{I}_{o}\right)-e A_{3} c_{2}\left(\partial_{2} \tilde{I}_{o}\right) \\
& \left.+c_{2} \beta\left(\partial_{3} \tilde{I}_{o}\right)^{2}\left(\partial_{2} \tilde{I}_{o}\right) e A_{3}\right]+e A_{0}\left[c_{3}\left(\partial_{0} \tilde{I}_{o}\right)+c_{3} \beta\left(\partial_{0} \tilde{I}_{o}\right)^{3}-c_{0} e A_{3}+c_{3} e A_{0}\right. \\
& \left.-c_{0}\left(\partial_{3} \tilde{I}_{o}\right)-c_{0} \beta\left(\partial_{3} \tilde{I}_{o}\right)^{3}+c_{3} e A_{0} \beta\left(\partial_{0} \tilde{I}_{o}\right)^{2}+c_{0} e A_{3} \beta\left(\partial_{3} \tilde{I}_{o}\right)^{2}\right] \\
& -F m^{2} c_{0}-Z m^{2} c_{3}=0 \text {. }
\end{aligned}
$$

Using the variables separation technique, the particle's action is defined as follows

$$
\tilde{I}_{o}=-\left(E-J \Omega_{H}\right) t+R(r)+N \chi+\Theta(\theta),
$$

where $J$ and $E$ denotes the angular momentum and particles energy, respectively and from the above Eqs.(9)-(12), we get a non-trivial matrix equation, i.e.,

$$
V\left(c_{0}, c_{1}, c_{2}, c_{3}\right)^{t}=0,
$$


where $V$ is a $4 \times 4$ matrix, whose elements are given as below

$$
\begin{aligned}
& V_{00}=-\dot{R}^{2} B D-B D \beta\left(E-J \Omega_{H}\right)^{4}-D C^{-1} N^{2}+D C^{-1} \beta N^{4}-\dot{\Theta}^{2} \\
& -\beta \dot{\Theta}^{2}-e A_{3} \dot{\Theta}-\beta e A_{3} \dot{\Theta}^{3}+m^{2} D-e A_{3} \dot{\Theta}+e^{2} A_{3}^{2} \beta \dot{\Theta}^{2}+e^{2} A_{3}^{2} \beta \dot{\Theta}^{3}, \\
& V_{01}=D B\left[\left(E-J \Omega_{H}\right) \dot{R}-\dot{R} \beta\left(E-J \Omega_{H}\right)^{3}+\dot{R} e A_{0}-\dot{R} \beta\left(E-J \Omega_{H}\right)^{2} e A_{0}\right] \\
& +B F\left[\dot{R} \dot{\Theta}+\beta \dot{R} \dot{T}^{3}-e A_{3} \dot{R}+e A_{3} \dot{\Theta} \dot{R}\right] \text {, } \\
& V_{02}=D C^{-1}\left[\left(E-J \Omega_{H}\right) N+\beta\left(E-J \Omega_{H}\right)^{3} N-e A_{0} N+\beta N e A_{0}\right. \\
& \left.\times\left(E-J \Omega_{H}\right)^{2}\right]+F C^{-1}\left[N \dot{T}-\beta \dot{\Theta}^{3} N-e A_{3} N+\beta e A_{3} \dot{\Theta}^{2} N\right], \\
& V_{03}=\beta e A_{0} N^{2} \dot{\Theta}-F C^{-1}\left[N^{2}-\beta \dot{\Theta}^{4}\right]+\left(E-J \Omega_{H}\right) \dot{\Theta}-\beta\left(E-J \Omega_{H}\right)^{3} \dot{\Theta} \\
& -e A_{0} \dot{\Theta}+e A_{3}\left[\left(E-J \Omega_{H}\right)-\beta\left(E-J \Omega_{H}\right)^{3}+e A_{0}+\beta\left(E-J \Omega_{H}\right)^{2}\right] \\
& \text { - } B D\left[\dot{R}^{2}-\beta \dot{R}^{3}\right]-m^{2} F \text {, } \\
& V_{10}=D\left[\left(E-J \Omega_{H}\right) \dot{R}+\beta\left(E-J \Omega_{H}\right) \dot{R}^{3}\right]+e A_{0} D\left[-\dot{R}+\beta \dot{R}^{3}\right] \\
& +e A_{3} F\left[-\dot{R}+\beta \dot{R}^{3}\right] \\
& V_{11}=D\left[\left(E-J \Omega_{H}\right)^{2}-\beta\left(E-J \Omega_{H}\right)^{4}+\left(E-J \Omega_{H}\right) e A_{0}+\beta e A_{0}\right. \\
& \left.\times\left(E-J \Omega_{H}\right)^{3}\right]+F\left[\left(E-J \Omega_{H}\right) \dot{\Theta}-\beta\left(E-J \Omega_{H}\right) \dot{\Theta}^{3}+\left(E-J \Omega_{H}\right) e A_{3}\right. \\
& \left.-\beta \dot{\Theta}^{2}\left(E-J \Omega_{H}\right) e A_{3}\right]+\left(Z D+F^{2}\right) C^{-1}\left[-N^{2}+\beta N^{4}\right]-m^{2} \\
& +e A_{0} D\left[-\left(E-J \Omega_{H}\right)+\beta\left(E-J \Omega_{H}\right)^{3}+e A_{0}-e A_{0} \beta\left(E-J \Omega_{H}\right)^{2}\right] \\
& +e A_{0} F\left[-\dot{\Theta}+\beta \dot{\Theta}^{3}-e A_{3}+\beta \dot{\Theta}^{2} e A_{3}\right]+e A_{3} A\left[-\dot{\Theta}+\beta \dot{\Theta}^{3}-e A_{3}\right. \\
& \left.-\beta \dot{\Theta}^{2} e A_{3}\right]+e A_{3} F\left[-\left(E-J \Omega_{H}\right)+\beta\left(E-J \Omega_{H}\right)^{3}+e A_{0}\right. \\
& \left.-\beta\left(E-J \Omega_{H}\right) e A_{0}\right] \text {, } \\
& V_{12}=\left(Z D+F^{2}\right) C^{-1}[\dot{R} N-\beta \dot{R} N] \text {, } \\
& V_{13}=F\left[-\left(E-J \Omega_{H}\right) \dot{R}+\beta\left(E-J \Omega_{H}\right) \dot{R}^{3}\right]-e A_{0} F\left[\dot{R}-\beta \dot{R}^{3}\right] \\
& +e A_{3} Z\left[\dot{R}-\beta \dot{R}^{3}\right] \text {, } \\
& V_{20}=D\left[-\left(E-J \Omega_{H}\right) N-\beta\left(E-J \Omega_{H}\right) N^{3}\right]-F\left[-N \dot{\Theta}+\beta \dot{\Theta} N^{3}\right] \\
& +e A_{0} D\left[N-\beta N^{3}\right]+e A_{3}\left[-N+\beta N^{3}\right] \\
& V_{21}=B\left[\dot{R} N-\beta \dot{R} N^{3}\right] \text {, } \\
& V_{22}=F\left[\left(E-J \Omega_{H}\right) \dot{\Theta}-\left(E-\Omega_{H} J\right) \beta \dot{\Theta}^{3}+e A_{0}\left(E-J \Omega_{H}\right)-\beta e A_{3}\right. \\
& \left.\times\left(E-J \Omega_{H}\right) \dot{\Theta}^{2}\right]+D\left[\left(E-J \Omega_{H}\right)^{2}-\beta\left(E-J \Omega_{H}\right)^{4}-e A_{0}\left(E-J \Omega_{H}\right)\right. \\
& \left.+\beta\left(E-J \Omega_{H}\right) N^{2}\right]-B\left[\dot{R}^{2}+\beta \dot{R}^{4}\right]+B\left[\dot{\Theta}^{2}+\beta \dot{\Theta}^{4}-e A_{3} \dot{\Theta}+\beta e A_{3} \dot{S}^{3}\right] \\
& +F\left[\left(E-J \Omega_{H}\right) \dot{\Theta}-\beta\left(E-J \Omega_{H}\right)^{3} \dot{\Theta}-e A_{0} \dot{\Theta}+\beta\left(E-J \Omega_{H}\right)^{2} \dot{\Theta} e A_{0}\right]
\end{aligned}
$$




$$
\begin{aligned}
&-\left(Z D+F^{2}\right) m^{2}+F e A_{0}\left[-\dot{\Theta}+\beta \dot{\Theta}^{3}-e A_{3}-\beta \dot{\Theta}^{2} e A_{3}\right] \\
&+D e A_{0}\left[-\left(E-J \Omega_{H}\right)+\beta\left(E-J \Omega_{H}\right)^{3}+e A_{0}+\beta e A_{0}\left(E-J \Omega_{H}\right)^{2}\right] \\
&+B e A_{3}\left[-\dot{S}+\beta \dot{S}^{3}-e A_{3}-\beta \dot{S}^{2} e A_{3}\right]+e A_{3}\left[-\left(E-J \Omega_{H}\right)\right. \\
&\left.+\beta\left(E-J \Omega_{H}\right)^{3}+e A_{0}+\beta e A_{0}\left(E-J \Omega_{H}\right)^{2}\right], \\
& V_{23}=F\left[\left(E-J \Omega_{H}\right) N+\beta\left(E-J \Omega_{H}\right) N^{3}\right]+\left[\dot{\Theta} N-\beta N^{3} \dot{\Theta}\right] B \\
&-e A_{0} F\left[\beta N^{3}-N\right]+e A_{3} B\left[\beta N^{3}-N\right], \\
& V_{30}=\left[-\dot{\Theta}-\beta\left(E-J \Omega_{H}\right) \dot{\Theta}^{3}+e A_{3}\left(E-J \Omega_{H}\right)-\beta e A_{3}\left(E-J \Omega_{H}\right) \dot{\Theta}^{2}\right] \\
&-B F\left[\dot{R}^{2}-\beta\left(E-J \Omega_{H}\right)^{3} \dot{R}+\beta \dot{R}^{4}\right]+F\left[-\dot{T} N+\beta N^{4}\right]+m^{2} F \\
&+e A_{0}\left[-\dot{\Theta}+\beta \dot{\Theta}^{3}-e A_{3}-\beta e A_{3} \dot{\Theta}^{2}\right], \\
& V_{31}=B F\left[-\left(E-J \Omega_{H}\right) \dot{R}+\beta\left(E-J \Omega_{H}\right)^{3} \dot{R}+e A_{0} \dot{\Theta}-\beta e A_{0}\left(E-J \Omega_{H}\right)^{2} \dot{\Theta}\right] \\
&-Z B\left[-\dot{R} \dot{\Theta}+\beta \dot{R} \dot{\Theta}^{2}-\dot{\Theta} e A_{3}-\beta \dot{\Theta}^{2} \dot{R} e A_{3}\right], \\
& V_{32}=F\left[-\left(E-J \Omega_{H}\right) \dot{\Theta}+\beta\left(E-J \Omega_{H}\right)^{3} \dot{\Theta}+e A_{0} \dot{\Theta}-\beta \dot{\Theta} e A_{0}\left(E-J \Omega_{H}\right)^{2}\right] \\
&+B\left[N \dot{\Theta}-\beta N \dot{\Theta}^{3}-N e A_{3}+\dot{\Theta}^{2} \beta N e A_{3}\right], \\
& V_{33}=\left[\left(E-J \Omega_{H}\right)^{2}-\beta\left(E-J \Omega_{H}\right)^{4}+e A_{3}+\beta\left(E-J \Omega_{H}\right)^{3} e A_{3}\right] \\
&-Z B\left[\dot{R}^{2}-\beta \dot{R}^{4}\right]-B\left[N^{2}+\beta N^{4}\right]-m^{2} Z+e A_{0}\left[-\left(E-J \Omega_{H}\right)\right. \\
&\left.+\beta\left(E-J \Omega_{H}\right)^{3}+e A_{0}-\beta e A_{0}\left(E-J \Omega_{H}\right)^{2}\right],
\end{aligned}
$$

where $\dot{R}=\partial_{r} \tilde{I}_{0}, \dot{\Theta}=\partial_{\theta} \tilde{I}_{0}$ and $N=\partial_{\chi} \tilde{I}_{0}$. For the non-trivial solution, we put $\operatorname{det}(\mathbf{V})=\mathbf{0}$ and computing the radial part for resultant components, the following integral can be obtained as follows

$$
R^{ \pm}= \pm \int \sqrt{\frac{\left(E-J \Omega_{H}-e A_{0}\right)^{2}+X_{2}\left(1+\frac{X_{1}}{X_{2}} \beta\right)}{B}} d r
$$

where $R^{-}$and $R^{+}$denote the radial function of incoming and outgoing boson particles, respectively. Although the function $X_{1}$ and $X_{2}$ can be determined as

$$
\begin{aligned}
X_{1} & =e A_{3} \frac{F}{D}\left[\left(E-J \Omega_{H}\right)^{3}-\left(E-J \Omega_{H}\right) e A_{0}\right]+\left(Z D+F^{2}\right)(C D)^{-1} N^{4} \\
& +\beta\left(E-J \Omega_{H}\right)^{3} e A_{0}+e A_{0}\left[\left(E-J \Omega_{H}\right)^{3}-e A_{0}\left(E-J \Omega_{H}\right)^{2}\right] \\
& -\frac{F}{D} \dot{\Theta}^{2}\left(E-J \Omega_{H}\right) e A_{3}+\frac{F}{D}\left(E-J \Omega_{H}\right) \dot{\Theta}^{3}-\left(E-J \Omega_{H}\right)^{4}+e A_{0} \frac{F}{D} \\
& \times\left[\dot{\Theta}^{3}+\dot{\Theta}^{2} e A_{3}\right]+e A_{3} Z\left[\dot{\Theta}^{3}-\dot{\Theta}^{2} e A_{3}\right],
\end{aligned}
$$

and

$$
\begin{aligned}
X_{2} & =\frac{F}{D}\left[\left(E-J \Omega_{H}\right) \dot{\Theta}+\left(E-J \Omega_{H}\right) e A_{3}\right]-\left(Z D+F^{2}\right)(C D)^{-1} N^{2}-m^{2} \\
& +e A_{0} \frac{F}{D}\left[-\dot{\Theta}-e A_{3}\right]-e A_{3} \frac{Z}{D} \dot{\Theta}-e A_{3}+e A_{3} \frac{F}{D}\left[-\left(E-J \Omega_{H}\right)+e A_{0}\right] .
\end{aligned}
$$

After applying taylor's series the functions $Z(r)$ and $B(r)$ near the horizon can be obtained as

$$
Z\left(r_{+}\right) \approx\left(r-r_{+}\right) Z^{\prime}\left(r_{+}\right), \quad B\left(r_{+}\right) \approx\left(r-r_{+}\right) B^{\prime}\left(r_{+}\right) .
$$

By using above relations in Eq.(15), we consider that the leading wave equation has two poles at $r=r_{+}$. Utilizing Eqs.(15) and (16), by integrating around the pole, we get

$$
\operatorname{Im} R^{ \pm}= \pm i \pi \frac{E-\Omega_{H} J-e A_{0}}{2 \kappa\left(r_{+}\right)(1+\Xi \beta)}
$$

where $\Xi=6\left(m^{2}+\frac{\left(J_{\theta}^{2}+J_{\phi}^{2} \csc ^{2} \theta\right)}{r_{+}^{2}}\right)>0$. 
The surface gravity $\kappa\left(r_{+}\right)$is given as follows [67]

$$
\begin{aligned}
\kappa\left(r_{+}\right) & =\left[\frac{\frac{l \alpha}{\omega}\left(\tilde{g}^{2}+\tilde{e}^{2}+\omega^{2} \tilde{k}\right)+\frac{\omega^{2} \tilde{k}}{a^{2}-l^{2}} r_{+}-M}{r_{+}^{2}+(l+a)^{2}} \times\left(\frac{(a-l) \alpha}{\omega} r_{+}+1\right)\right. \\
& \left.\times\left(1-\frac{(a+l) \alpha}{\omega} r_{+}\right)\right] .
\end{aligned}
$$

The corrected tunneling probability $(\Gamma)$ for boson particles can be obtained as

$$
\begin{aligned}
\Gamma & =\frac{\operatorname{Prob}[\text { emission }]}{\operatorname{Prob}[\text { absorption }]}=\frac{\exp \left[-2\left(\operatorname{Im} R^{+}+\operatorname{Im} \Theta\right)\right]}{\exp \left[-2\left(\operatorname{Im} R^{-}-\operatorname{Im} \Theta\right)\right]}=\exp \left[-4 \operatorname{Im}^{+}\right], \\
& =\exp \left[\frac{-2 \pi\left(E-J \Omega_{H}-e A_{0}\right)(1+\beta \Xi)}{\frac{\frac{\alpha l}{\omega}\left(\tilde{g}^{2}+\tilde{e}^{2}+\omega^{2} \tilde{k}\right)-M+\frac{\omega^{2} \tilde{k}}{a^{2}-l^{2}} r_{+}}{r_{+}^{2}+(l+a)^{2}} \times\left(1-\frac{\alpha(l-a)}{\omega} r_{+}\right) \times\left(1-\frac{\alpha(l+a)}{\omega} r_{+}\right)}\right] .
\end{aligned}
$$

We calculate the corrected Hawking temperature by comparing the tunneling probability with Boltzmann factor, i.e., $\Gamma_{B}=e^{-\left(E-J \Omega_{H}-e A_{0}\right) / T_{H}^{\prime}}$

$$
\begin{aligned}
T_{H}^{\prime} & =\left[\frac{\left(\frac{l \alpha}{\omega}\left(\tilde{g}^{2}+\tilde{e}^{2}+\omega^{2} \tilde{k}\right)+\frac{\omega^{2} \tilde{k}}{a^{2}-l^{2}} r_{+}-M\right)\left(1-\frac{(l-a) \alpha}{\omega} r_{+}\right)\left(1-\frac{(l+a) \alpha}{\omega} r_{+}\right)}{2 \pi\left(r_{+}^{2}+(l+a)^{2}\right)(1+\Xi \beta)}\right], \\
T_{H}^{\prime} & =\left[\frac{\left(\frac{l \alpha}{\omega}\left(\tilde{g}^{2}+\tilde{e}^{2}+\omega^{2} \tilde{k}\right)+\frac{\omega^{2} \tilde{k}}{a^{2}-l^{2}} r_{+}-M\right)\left(1-\frac{(l-a) \alpha}{\omega} r_{+}\right)\left(1-\frac{(l+a) \alpha}{\omega} r_{+}\right)}{2 \pi\left(r_{+}^{2}+(l+a)^{2}\right)}\right] \\
& \times\left[1-\beta \Xi+(\beta \Xi)^{2}+\ldots\right],
\end{aligned}
$$

by considering only first order quantum corrections, we can write

$$
T_{H}^{\prime}=T_{H}[1-\beta \Xi]
$$

where the semi-classical Hawking temperature $T_{H}$ is given as follows

$$
T_{H}=\left[\frac{\left(\frac{l \alpha}{\omega}\left(\tilde{g}^{2}+\tilde{e}^{2}+\omega^{2} \tilde{k}\right)+\frac{\omega^{2} \tilde{k}}{a^{2}-l^{2}} r_{+}-M\right)\left(1-\frac{(l-a) \alpha}{\omega} r_{+}\right)\left(1-\frac{(l+a) \alpha}{\omega} r_{+}\right)}{2 \pi\left(r_{+}^{2}+(l+a)^{2}\right)}\right] .
$$

The corrected tunneling probability depends on $A_{0}, E, \ell, \omega, e, \alpha, J$ and $\beta$, i.e., vector potential of BHs, energy of particle, NUT parameter, kerr-like rotation parameter, charges of particles, acceleration of BHs, angular momentum of particle and correction parameter, respectively. We can observe that the corrected Hawking temperature does not only depend upon the $\mathrm{BH}$ properties but also depends upon the mass and angular momentum of the radiated particles and quantum corrections $\beta$. It is to be noted that the first order correction term is same as semi-classical original Hawking term $T_{H}$, while the next order correction term must be smaller than the preceding term satisfying GUP.

It is to be noted that the corrected temperature of boson particles given in Eq.(18) reduced to the temperature of fermion particles in Eq.(4.20) for $(\beta=0)$ of Ref.[67]. So, the corrected temperature depends upon the correction parameter. Also, for $\ell=0$ and $\tilde{k}=1$, the above result reduces to the Hawking temperature of accelerating and rotating BHs with electric and magnetic charges [83]. For $\alpha=0$, we recover the temperature for non-accelerating BHs [84]. Moreover, for $\beta=0, \ell=0, \tilde{k}=1 \& \alpha=0$ in Eq.(18), the Hawking temperature of the Kerr-Newman $\mathrm{BH}[85]$ is recovered and which is reduced for $a=0$ to the temperature of ReissnerNordström $\mathrm{BH}$, for $Q=0$, the temperature exactly reduces to the Hawking temperature of the Schwarzschild $\mathrm{BH}$ [86]. In order to calculate the residual mass of $\mathrm{BH}$, the temperature can be expressed as

$$
T_{H}^{\prime}=\frac{1}{8 \pi M}\left[1-6 \beta\left(m^{2}+\frac{\left(J_{\theta}^{2}+J_{\phi}^{2} \csc ^{2} \theta\right)}{r_{+}^{2}}\right)\right],
$$


where $\left(m^{2}+\frac{\left(J_{\theta}^{2}+J_{\phi}^{2} \csc ^{2} \theta\right)}{r_{+}^{2}}\right)$ denotes the kinetic energy component of radiated particles related with tangent plane at horizon. For residual mass, we approximate the kinetic energy component as $\omega^{2}$. Quantum corrections decelerate the increase in temperature during the radiation process. These corrections cause the radiation ceased at some specific temperature, leaving the remnant mass. The temperature stops increasing when this condition holds [70]

$$
(M-d M)(1+\beta \Xi) \simeq M
$$

For $d M=\omega, \beta=\frac{\beta_{0}}{M_{p}^{2}}$ and $\omega \simeq M_{p}$ where $M_{p}$ is the Planck mass and $\beta_{0}$ is a dimensionless parameter representing quantum gravity effects and $\beta_{0}<10^{34}$, one can obtain the following constraints

$$
M_{\text {Res }} \simeq \frac{M_{p}^{2}}{\beta_{0} \omega} \gtrsim \frac{M_{p}}{\beta_{0}}, \quad T_{\text {Res }} \lesssim \frac{\beta_{0}}{8 \pi M_{p}}
$$

It is important to mention here that the value of the corrected Hawking temperature is smaller than the original temperature and $\mathrm{BH}$ stops radiating, when the mass of the $\mathrm{BH}$ reaches to its minimal value $M_{\text {Res }}$.

\section{A. Graphical Analysis of $T_{H}^{\prime}$ versus $r_{+}$}

This subsection is devoted to study the graphical behavior of Hawking temperature $T_{H}^{\prime}$ with respect to horizon $r_{+}$ under quantum gravity effects. Furthermore, we study the physical significance of these graphs under the influence of correction parameter $\beta, N U T$ parameter $\ell$, rotation parameters $a$ and $\omega, \mathrm{BH}$ acceleration $\alpha$, electric and magnetic charges $e$ and $g$, arbitrary parameter $k$, for fixed BH mass $M=1$ and arbitrary parameter $\Xi=0.01$. We analyze stability and instability of accelerating and rotating $\mathrm{BH}$ associated with NUT parameter.

Figure 1 indicates the behavior of $T_{H}^{\prime}$ w.r.t $r_{+}$for fixed $\alpha=0.1, k=0.5$ and $\ell=0.4$.

(i) There can be seen an exponential increase in the temperature $T_{H}^{\prime}$ and after attaining a height the $T_{H}^{\prime}$ slightly decreases with the increasing horizon $r_{+}$for varying values of rotation parameter $a$. The $T_{H}^{\prime}$ decreases as horizon increases, this physical behavior reflects the $\mathrm{BH}$ stability with positive temperature till $r_{+} \rightarrow \infty$. The temperature increases with the increase of $a$.

(ii) We observe the behavior of temperature for varying values of correction parameter $\beta$ with fixed values of different parameters. The $T_{H}^{\prime}$ decreases with increasing horizon in the domain $0 \leq r_{+} \leq 15$ after attaining a maximum height. The maximum temperature with non-zero horizon reflects the $\mathrm{BH}$ remnant. This physical behavior of $T_{H}^{\prime}$ indicates the $\mathrm{BH}$ stability with positive range. It is also worthy to note that the $T_{H}^{\prime}$ increases with the increase of correction parameter $\beta$.

Figure 2 shows the behavior of $T_{H}^{\prime}$ w.r.t $r_{+}$for fixed $\alpha=0.1, a=0.5 \& \ell=0.4$.

(i) Figure shows that the $T_{H}^{\prime}$ exponentially increases and slowly falls down from a height for different values of electric and magnetic charges. The decrease in $T_{H}^{\prime}$ with the increasing horizon exhibits the stable state of $\mathrm{BH}$ in the domain $0 \leq r_{+} \leq 15$. It can be seen that the $T_{H}^{\prime}$ decreases with the increase of $\mathrm{BH}$ electric and magnetic charges $e$ and $g$, respectively.

(ii) Figure indicates that the temperature eventually drops down from a height for different values of arbitrary parameter $k$. There is a significant change in temperature as it decreases exponentially and attains an asymptotically flat shape which indicates BH stability till $r_{+} \rightarrow+\infty$. The $T_{H}^{\prime}$ increases with increase of $k$.

Figure 3 depicts the behavior of $T_{H}^{\prime}$ w.r.t $r_{+}$for fixed $a=0.5, e=g=5 \& \ell=0.4$ for varying $a$ and $\omega$ in the domain $0 \leq r_{+} \leq 5$.

(i) We can observe that initially the temperature increases with increasing horizon and after a maximum height it exponentially decreases with increasing which indicates the stable state of $\mathrm{BH}$ for different values of $\mathrm{BH}$ acceleration $\alpha$.

(ii) There can be seen that $T_{H}^{\prime}$ exponentially increases and eventually falls down from a height and decreases as horizon increases till $r_{+} \rightarrow+\infty$. This physical behavior identify the stability of BH with positive range for different values of $\omega$. We can observe that with the increase in the value of $\omega$ the $T_{H}^{\prime}$ increases.

\section{CONCLUSION AND DISCUSSION}

In this paper, we have studied the quantum gravity effects for spin-1 (boson) particles from charged accelerating rotating BH having NUT parameter. For this purpose, by considering the GUP effects, we have used modified Lagrangian equation incorporating quantum effects described the motion of spin-1 particles. Later, by applying the 
Peer-reviewed version available at Chinese Physics C 2020; doi:10.1088/1674-1137/44/1/015104

(i) $\beta=1, e=g=0.2, \omega=0.1$

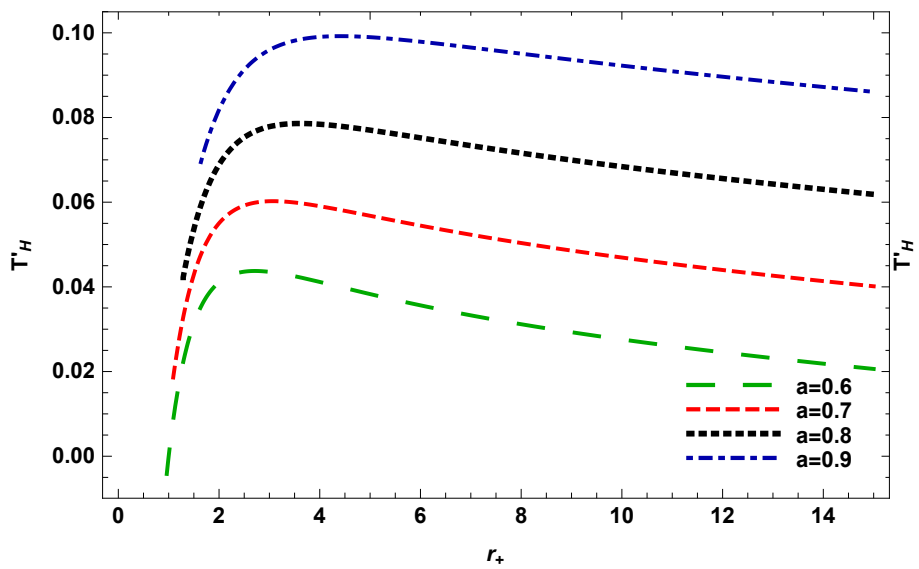

(ii) $\mathrm{a}=0.5, \mathrm{e}=\mathrm{g}=0.1, \omega=0.05$

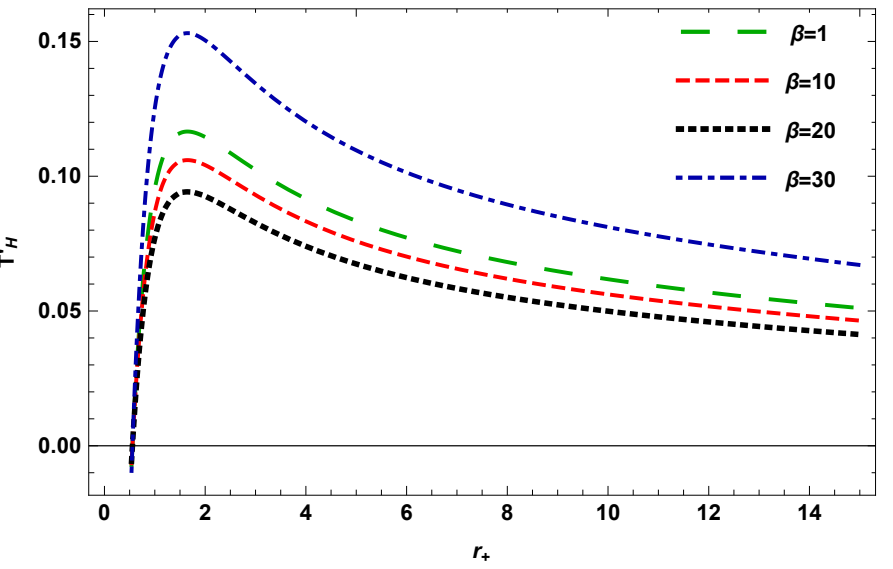

FIG. 1: $T_{H}^{\prime}$ vs $r_{+}$for $\alpha=0.1, k=0.5 \& \ell=0.4$.

(i) $\omega=0.1, \beta=1, k=0.1$

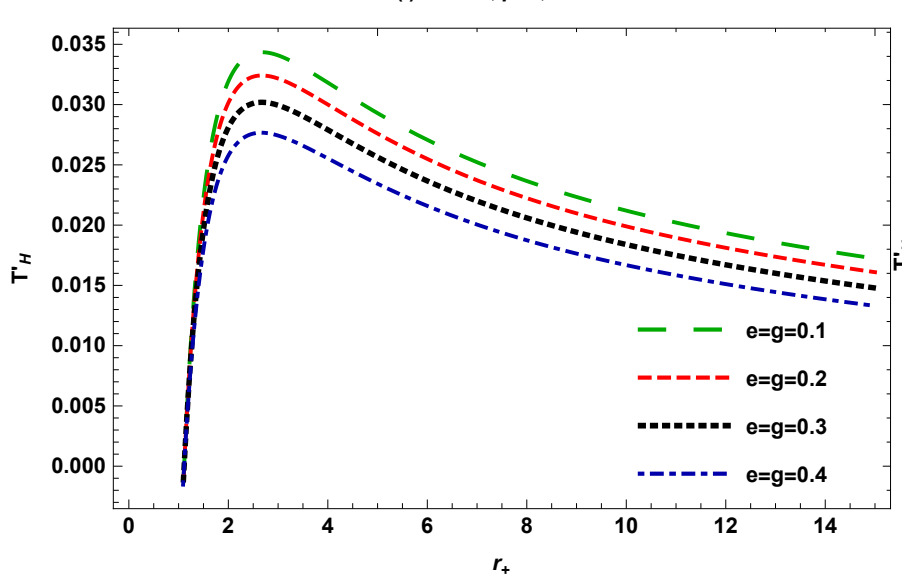

(ii) $\omega=3, \beta=1, e=g=0.1$

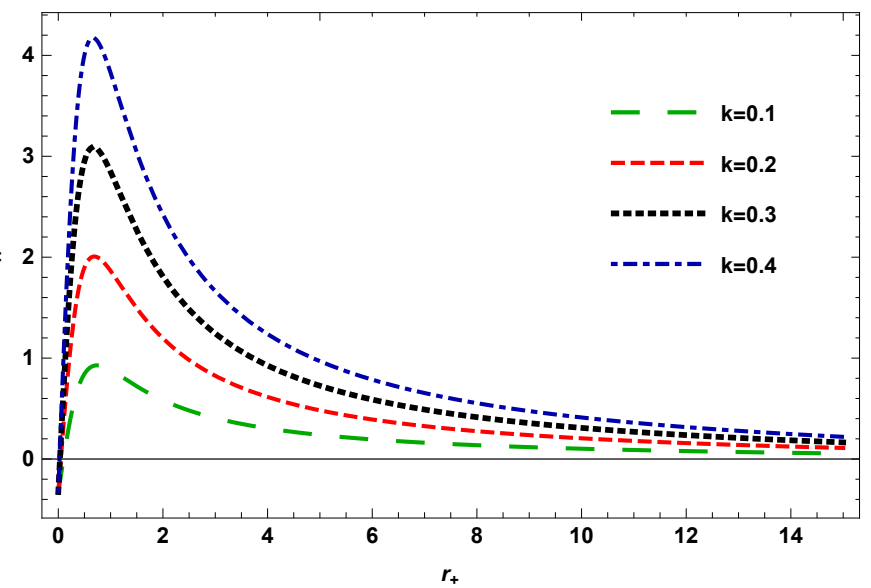

FIG. 2: $T_{H}^{\prime}$ vs $r_{+}$for $\alpha=0.1, a=0.5, \& \ell=0.4$.

(i) $\omega=1.5, \beta=10, k=0.4$

(ii) $\alpha=0.1, \beta=0.5, k=0.5$
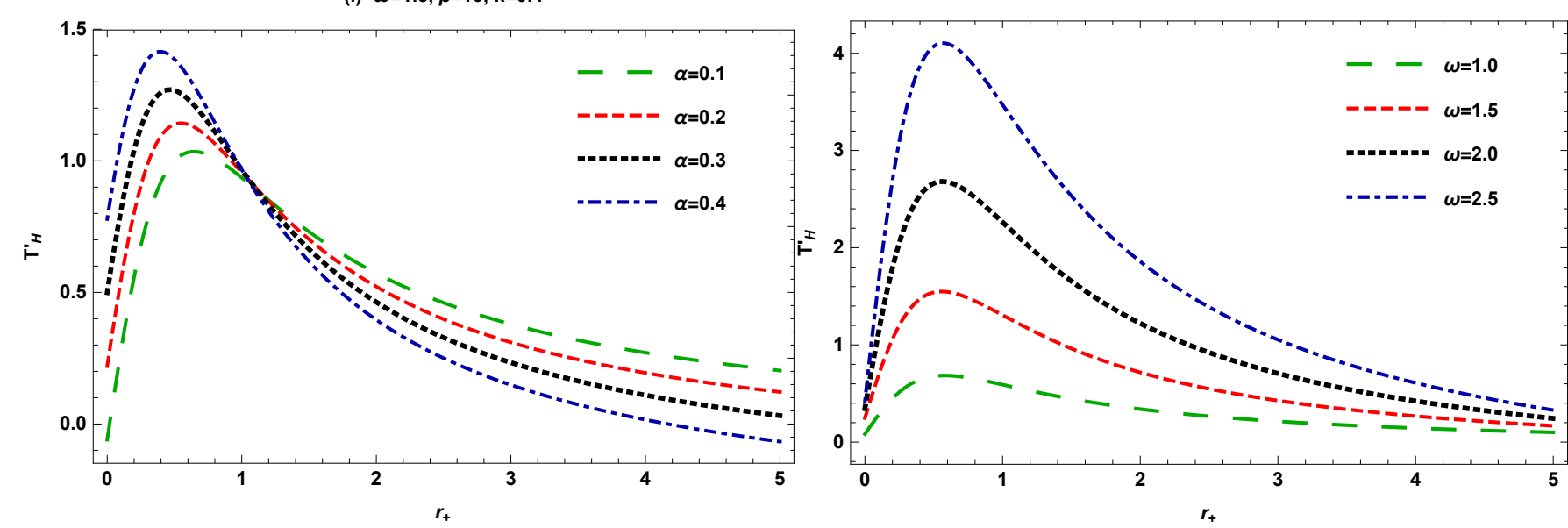

FIG. 3: $T_{H}^{\prime}$ vs $r_{+}$for $a=0.5, e=g=5 \& \ell=0.4$. 
Hamilton-Jacobi technique, we have calculated the tunneling probabilities of boson particles. Moreover, we have analyzed the corrected Hawking temperatures of these BHs. We have concluded that the modified tunneling probabilities are not just depend upon the BHs properties but also depend on the properties of emitted boson particles, i.e., energy, potential, surface gravity, particles charge and total angular momentum. Moreover, it is important to note that the modified tunneling probabilities as well as Hawking temperature depend on the quantum particles which contributes gravitational radiation in form of massive particles (BH's energy carrier) tunneling.

When the quantum gravity effects are neglected, i.e., $(\beta=0)$, then the corrected Hawking temperature Eq.(18) is reduced to the absolute temperature obtained by quantum tunneling of boson (spin-1) particles [75]. If we ignore the potential effects $(A=0)$, the modified Hawking temperature reduced to the temperature of vector (spin-1) particles provided in Refs.[66,87]. Also, for $\ell=0$ and $\tilde{k}=1$, the above results are reduced to the Hawking temperature of the accelerating and rotating BHs with electric and magnetic charges [83]. For $\alpha=0$, we have recovered the temperature of non-accelerating BHs from the Hawking temperature of the charged accelerating and rotating BHs [84]. Moreover, for $\beta=0, \ell=0, \tilde{k}=1$ and $\alpha=0$ in Eq.(18), the Hawking temperature of the Kerr-Newman BH [85] is recovered, which is reduced for $a=0$ to the temperature of the RN BH. For $Q=0$, the temperature exactly reduces to the Hawking temperature of the Schwarzschild $\mathrm{BH}$ at the residual mass of the $\mathrm{BH}$ [86].

In our analysis we have found that the quantum corrections decelerate the increase in temperature during the radiation process. This correction causes the radiation ceased at some specific temperature, leaving the remnant mass. The remnant will obtain at specific condition

$$
M_{\text {Res }} \simeq \frac{M_{p}^{2}}{\beta_{0} \omega} \gtrsim \frac{M_{p}}{\beta_{0}} .
$$

It is important to mention here that the value of the corrected Hawking temperature is smaller than the original Hawking temperature and it stops radiating, when the mass of the $\mathrm{BH}$ reaches to its minimal value $M_{\text {Res }}$. However, this result is still hold if background $\mathrm{BH}$ geometry is more generalized.

The results from the graphical analysis of corrected Hawking temperatures with respect to the horizon for the given $\mathrm{BH}$ is summarized as follows:

- For accelerating and rotating BH with NUT parameter, the $T_{H}^{\prime}$ decreases with the increasing horizon and $\mathrm{BH}$ reflects the stable state for varying values of rotation parameter $a$ and correction parameter $\beta$. The corrected temperature $T_{H}^{\prime}$ also increases with the increase in $a$ and $\beta$. The $\mathrm{BH}$ remnant can be obtained at nonzero horizon with maximum temperature for different values of $\beta$ in the domain $0 \leq r_{+} \leq 15$.

- The corrected temperature $T_{H}^{\prime}$ decreases with the increase of $e$ and $g$. For different values of electric and magnetic charges, the $\mathrm{BH}$ reflects stability in the domain $0 \leq r_{+} \leq 15$.

- The $T_{H}^{\prime}$ increases with the increase in the value of arbitrary parameter $k$.

- The $T_{H}^{\prime}$ increases with the increase in $\mathrm{BH}$ acceleration $\alpha$ and rotation parameter $\omega$.

- In our analysis, we have considered the value $\Xi=0.01$, then the condition of GUP must be satisfied for arbitrary values of $0 \leq \beta<100$, the correction term is smaller than the usual term as well as positive temperature is obtained. While, for $\beta>100$ the first order correction term becomes greater than the usual term and the condition of GUP do not satisfy and we observe the negative temperature which is non-physical. Furthermore, for $\beta=100$, the semi-classical term cancel out with first order correction term and hence the temperature vanishes.

\section{Acknowledgments}

A. Ö. acknowledges financial support provided under the Chilean FONDECYT Grant No. 3170035.

[1] Hawking, S.W.: Nature 248(1974)30.

[2] R. Kerner, R.B. Mann, Class. Quant. Grav. 25, 095014(2008). ibid; Phys. Rev. D 73, 104010(2006).

[3] Q.Q. Jiang, S.Q. Wu, X. Cai, Phys. Rev. D 73, 064003(2006).

[4] H. Erbin, V. Lahoche, Phys. Rev. D 98, 104001(2018).

[5] M. K. Parikh, F. Wilczek, Phys. Rev. Lett. 85, 5042(2000).

[6] K. Srinivasan, T. Padmanabhan, Phys. Rev. D 60, 024007(1999). 
[7] R. Banerjee, B. R. Majhi, J. High Energy Phys. 06, 095(2008).

[8] L. Brillouin, Comptes Rendus de 1Academie des Sciences 183, 24(1926);

[9] I. Sakalli and A. Övgün, Gen. Rel. Grav. 48, no. 1, 1 (2016).

[10] I. Sakalli and A. Ovgun, Eur. Phys. J. Plus 130, no. 6, 110 (2015).

[11] I. Sakalli and A. Ovgun, EPL 110, no. 1, 10008 (2015).

[12] I. Sakalli and A. Ovgun, EPL 118, no. 6, 60006 (2017).

[13] I. Sakalli and A. Ovgun, Astrophys. Space Sci. 359, no. 1, 32 (2015).

[14] I. Sakalli and A. Ovgun, J. Exp. Theor. Phys. 121, no. 3, 404 (2015).

[15] I. Sakalli and A. Övgün, Eur. Phys. J. Plus 131, no. 6, 184 (2016).

[16] A. Övgün, Int. J. Theor. Phys. 55, no. 6, 2919 (2016).

[17] A. Övgün and K. Jusufi, Eur. Phys. J. Plus 132, no. 7, 298 (2017)

[18] K. Jusufi, A. Ovgun and G. Apostolovska, Adv. High Energy Phys. 2017, 8798657 (2017)

[19] X. M. Kuang, J. Saavedra and A. Övgün, Eur. Phys. J. C 77, no. 9, 613 (2017).

[20] X. M. Kuang, B. Liu and A. Övgün, Eur. Phys. J. C 78, no. 10, 840 (2018)

[21] W. Javed, R. Babar and A. Övgün, Mod. Phys. Lett. A 34, no. 09, 1950057 (2019)

[22] P. A. Gonzalez, A. Övgün, J. Saavedra and Y. Vasquez, Gen. Rel. Grav. 50, no. 6, 62 (2018).

[23] A. Övgün, Adv. High Energy Phys. 2017, 1573904 (2017).

[24] K. Jusufi and A. Övgün,, Int. J. Theor. Phys. 56, no. 6, 1725 (2017).

[25] Deyou Chen, Houwen Wu, Haitang Yang, Shuzheng Yang, Int. J. Mod. Phys. A 29 (2014) 1430054.

[26] Deyou Chen, Eur. Phys. J. C 74 (2014) 2687.

[27] G. Gecim and Y. Sucu, Mod. Phys. Lett. A 33, no. 28, 1850164 (2018).

[28] G. Gecim and Y. Sucu, Phys. Lett. B 773, 391 (2017).

[29] G. Gecim and Y. Sucu, Adv. High Energy Phys. 2018, 8728564 (2018).

[30] W. Javed, R. Ali, R. Babar and A. Övgün, Preprints 2019, 2019030194 (doi: 10.20944/preprints201903.0194.v3).

[31] M. Hossain Ali and K. Sultana, Int.J.Theor.Phys. 56, no.7, 2279-2292 (2017).

[32] M. Hossain Ali, Class.Quant.Grav. 24, 5849-5860 (2007).

[33] M. Hossain Ali, Int.J.Theor.Phys. 47, 2203-2217 (2008).

[34] M. Hossain Ali, Gen.Rel.Grav. 36, 1171-1181 (2004).

[35] H. Pasaoglu and I. Sakalli, Int. J. Theor. Phys. 48, 3517 (2009).

[36] E. T. Akhmedov, V. Akhmedova and D. Singleton, Phys. Lett. B 642, 124 (2006).

[37] V. E. Akhmedova, T. Pilling, A. de Gill and D. Singleton, Theor. Math. Phys. 163, 774 (2010).

[38] A. de Gill, D. Singleton, V. Akhmedova and T. Pilling, Am. J. Phys. 78, 685 (2010)

[39] T. Zhu, J. R. Ren and D. Singleton, Int. J. Mod. Phys. D 19, 159 (2010)

[40] V. Akhmedova, T. Pilling, A. de Gill and D. Singleton, Phys. Lett. B 666, 269 (2008)

[41] V. Akhmedova, T. Pilling, A. de Gill and D. Singleton, Phys. Lett. B 673, 227 (2009)

[42] E. T. Akhmedov, V. Akhmedova, T. Pilling and D. Singleton, Int. J. Mod. Phys. A 22, 1705 (2007)

[43] S. Kanzi and I. Sakalli, arXiv:1905.00477 [hep-th].

[44] M. Rizwan, M. Z. Ali and A. Övgün, doi:10.1142/S0217732319501840, arXiv:1812.01983 [physics.gen-ph].

[45] G. Gecim and Y. Sucu, Gen. Rel. Grav. 50, no. 12, 152 (2018)

[46] I. A. Meitei, T. I. Singh, S. G. Devi, N. P. Devi and K. Y. Singh, Int. J. Mod. Phys. A 33, no. 12, 1850070 (2018).

[47] G. R. Chen, S. Zhou and Y. C. Huang, Int. J. Mod. Phys. D 24, no. 01, 1550005 (2014)

[48] X. X. Zeng and S. Z. Yang, Chin. Phys. B 18, 462 (2009).

[49] S. K. Modak, Phys. Lett. B 671, 167 (2009)

[50] R. Li and J. R. Ren, Phys. Lett. B 661, 370 (2008)

[51] H. L. Li and S. Z. Yang, EPL 79, no. 2, 20001 (2007).

[52] Q. Q. Jiang, S. Q. Wu and X. Cai, Phys. Lett. B 651, 58 (2007)

[53] X. He and W. Liu, Phys. Lett. B 653, 330 (2007).

[54] T. M. He and J. Y. Zhang, Chin. Phys. Lett. 24, 3336 (2007).

[55] H. L. Li, S. Z. Yang, Q. Q. Jiang and D. J. Qi, Phys. Lett. B 641, 139 (2006).

[56] X. Q. Li, G.R. Chen, Phys. Lett. B 751, 34(2015).

[57] G. R. Chen, Y.C. Huang, Int. J. Mod. Phys. Rev. A 30, 1550083(2015).

[58] Z. Feng, Y. Chen, X. Zu, Astrophys. space Sci. 48, 359(2015).

[59] K. Jusufi, Ali Övgün, Astrophys. Space Sci. 361, 207(2016).

[60] J. M. Bardeen, in Conference Proceedings of GR5 (Tbilisi, URSS, 1968), p. 174.

[61] A.D. Vries, T.S. Kaler, Phys. Rev. D 65, 104022(2002).

[62] Q.Q. Jiang, Phys. Rev. D 78, 044009(2008).

[63] T. Jian, C. Bing-Bing, Acta Physica Polonica, 40, 241(2009).

[64] A. Yale, Phys. Lett. B 697, 398(2011).

[65] M. Sharif, W. Javed, Can. J. Phys. 90, 903(2012); ibid. Gen. Relativ. Gravit. 45, 1051(2013); ibid. J. Exp. Theor. Phys. 115, 782(2012); ibid. Proceedings of the 3rd GalileoXu Guangqi Meeting, Int. J. Mod. Phys.: Conference Series, 23, 271(2013);ibid. Proceedings of the 13th Marcel Grossmann Meeting (Stockholm, 2012), World Scientific, 3, 1950(2015); ibid. J. Korean. Phys. Soc. 57, 217(2010).

[66] M. Sharif, W. Javed, Can. J. Phys. 91, 43(2013).

[67] M. Sharif, W. Javed, Eur. Phys. J. C 72, 1997(2012). 
[68] A. Övgün, W. Javed, R. Ali, Advances in High Energy Physics, 3131620, 11(2018).

[69] M.A Anacleto, F.A. Brito, G.C Luna, E. Passos, Annals of Phys. 362, 436(2015).

[70] G. Li, X. Zu, Journal of Applied Mathematics and Physics 3, 134(2015).

[71] M.A. Anacleto, F.A Brito, E. Passos, Phys. Lett. B 749, 81(2015).

[72] A. Övgün, K. Jusufi, Eur. Phys. J. Plus 131, 177(2016).

[73] T.I. Singh, I.A. Meitei, K.Y. Singh, Astrophys Space Sci 103, 361(2016).

[74] I. Sakalli, A. Övgün, K. Jusufi, Astrophys Space Sci 361, 330(2016).

[75] W. Javed, G. Abbas, R. Ali, Eur. Phys. J. C 77, 296(2017).

[76] W. Chen, H. Lü, C. N. Pope, Class. Quantum Gravity 23, 5323(2006); ibid. Nucl. Phys. B 762, 38(2007).

[77] J.B. Griffiths, J. Padolsky, Class. Quant. Gravity 24, 1687(2007).

[78] A.A. Badawi, M. Halilsoy, Gen. Relativ. Gravity 38, 1729(2006).

[79] J. B. Griffiths, J. Padolsky, Class. Quant. Gravity 22, 3467(2005).

[80] X.Q. Li, G.R. Chen, Phys. Lett. B 751, 34(2015).

[81] X.Q. Li, Phys. Lett. B 763, 80(2016).

[82] T. Shivalingaswamy, B.A. Kagali, Eur. J. Phys. Education 2, 1309(2011).

[83] U.A. Gillani, K. Saifullah, Phys. Lett. B699, 15(2011); U.A. Gillani, M. Rehman, and K. Saifullah, JCAP 06, 016(2011); M. Rehman, and K. Saifullah, JCAP 03, 001(2011).

[84] M. Bilal, K. Saifullah, arXiv:1010.5575.

[85] R. Kerner, R.B. Mann, Phys. Lett. B665, 277(2008).

[86] D.Y. Chen, Q.Q. Jiang, X.T. Zua, Phys. Lett. B665, 106(2008).

[87] M. Cvetič, S.S. Gubser, JHEP 04, 024(1999). 